\title{
Roles of hsa-miR-12462 and SLC9A1 in acute myeloid leukemia
}

\author{
Yan Jia ${ }^{1 \dagger}$, Wei Liu ${ }^{1 \dagger}$, Hui-En Zhan ${ }^{2}$, Xiao-Ping Yi ${ }^{3}$, Hui Liang ${ }^{1}$, Qi-Li Zheng ${ }^{1}$, Xin-Ya Jiang ${ }^{1}$, Hai-Yan Zhou ${ }^{4}$, \\ Liang Zhao ${ }^{1}$, Xie-Lan Zhao ${ }^{1}$ and Hui Zeng ${ }^{2^{*}}$ (I)
}

\begin{abstract}
MicroRNAs (miRNAs) play important roles in cell proliferation, differentiation, and survival and may be useful for acute myeloid leukemia (AML) diagnosis and prognosis. In this study, we defined a novel miRNA, hsa-miR-12462, through small RNA sequencing of the bone marrow (BM) cells from 128 AML patients. Overexpression of hsa-miR12462 in AML cells (U937 and HL-60) significantly decreased their growth rate when compared with those of the wild-type and MOCK controls. In a xenograft mouse model, tumor weight and size in the mice bearing the U937 cells with hsa-miR-12462 overexpression were significantly reduced when compared with those bearing the mock cells. The AML cells overexpressing hsa-miR-12462 had increased sensitivity to cytarabine chemotherapy. Combining the data from the MiRDB, an online microRNA database (http://mirdb.org), with the RNA-sequencing results, SLC9A1 was predicted to be one of the targets of hsa-miR-12462. hsa-miR-12462 was further confirmed to bind exclusively to the 3'UTR of SLC9A1 in U937 cells, leading to downregulation of SLC9A1. In summary, a higher level of hsa-miR12462 in AML cells is associated with increased sensitivity to cytarabine chemotherapy via downregulation of SLC9A1.
\end{abstract}

Keywords: miRNA, Acute myeloid leukemia, SLC9A1, RNA sequencing

To the Editor,

MicroRNAs (miRNAs) play important roles in cell proliferation, differentiation, and survival and may be useful for acute myeloid leukemia (AML) diagnosis and prognosis [1-4]. In this study, we defined a novel miRNA, hsamiR-12462, through small RNA sequencing of the bone marrow (BM) cells from 128 newly diagnosed subjects with AML (Supplementary Table1-2). Based on 2016 World Health Organization (WHO) criteria, all subjects were grouped into 2 cohorts: (1) those achieving a complete remission (CR) with conventional induction chemotherapy and remaining in $\mathrm{CR} \geq 6$ months and (2) those not achieving $\mathrm{CR}$ after 2 courses of standard

\footnotetext{
* Correspondence: androps2011@hotmail.com

${ }^{\dagger}$ Yan Jia and Wei Liu are co-first authors.

${ }^{2}$ Department of Hematology, The First Affiliated Hospital of Jinan University, Guangzhou, Guangdong, China

Full list of author information is available at the end of the article
}

induction chemotherapy (refractory) or relapsed in $<6$ months after CR (relapsed) [5]. Small RNA sequencing of these samples revealed different miRNA expression profiles between CR and refractory/relapsed (RR) AML patients [6]. One miRNA showed the highest differential expression pattern in this analysis. This miRNA has never been reported in the literature. We named this miRNA as hsa-miR-12462 (Figure S1A). Next, we explored the biological activity of hsa-miR-12462 by overexpressing it in AML cells using a lentiviral vector (Figure S1B). The growth rate of the hsa-miR-12462 overexpressing cells was significantly decreased when compared with those of the wild-type and MOCK controls in both U937 and HL60 cells (Fig. 1a, Figure S1C). U937 cells were confirmed using an EdU incorporation assay (Figure S1D-E).

Treatment of AML cells with cytarabine for up to $48 \mathrm{~h}$ resulted in a lower proliferation of the hsa-miR-12462 overexpressing cells when compared with that of the 


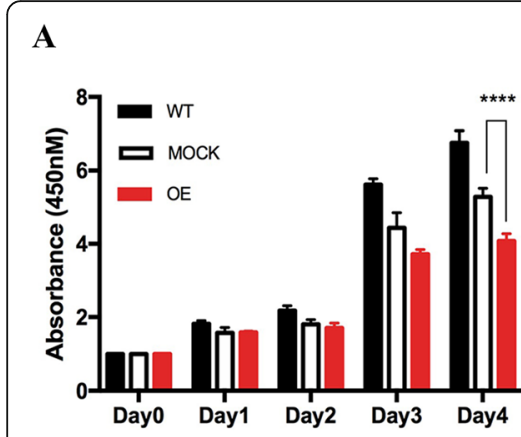

D

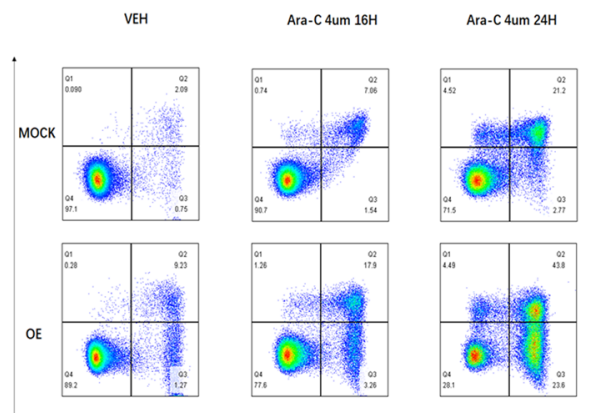

G

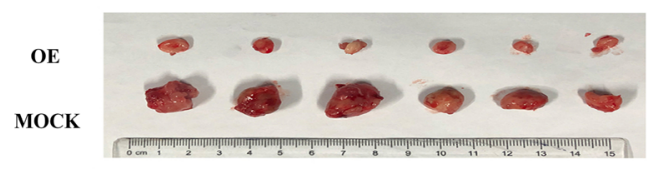

$\mathbf{H}$
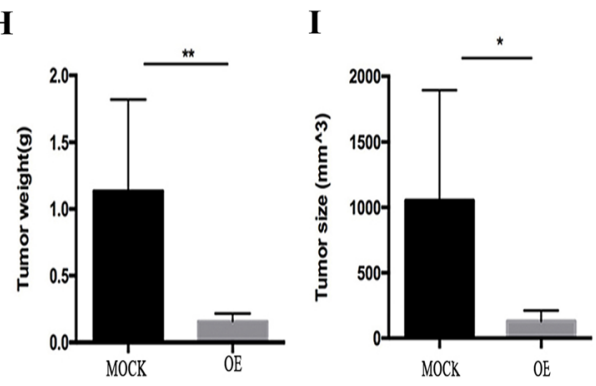

B

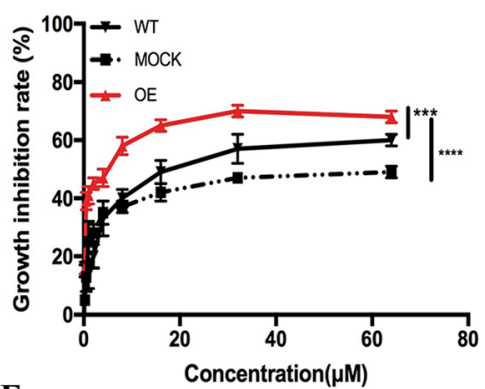

$\mathbf{E}$

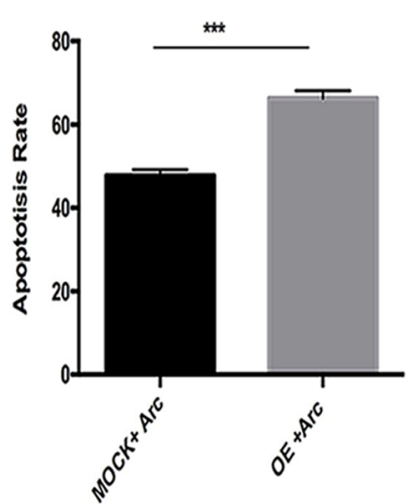

C

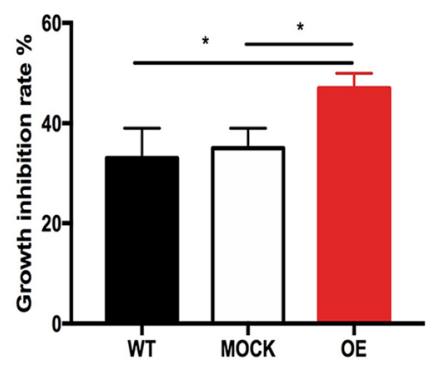

F

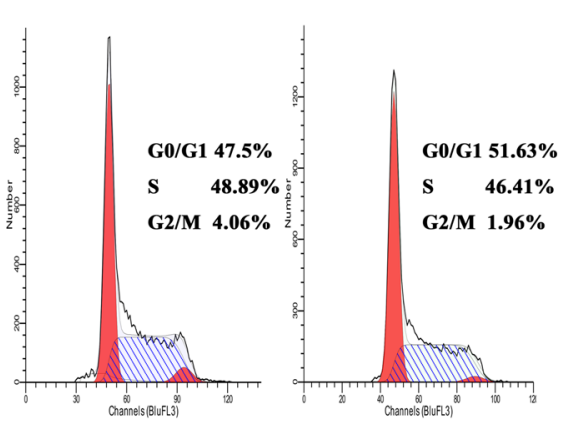

Fig. 1 Overexpression of hsa-miR-12462 inhibits the growth of U937 cells and increases cytarabine sensitivity. a Growth rates of U937 wild-type, MOCK-infected, and overexpressing cells (OE vs. MOCK $P<0.0001$ ). b Growth inhibition of wild-type, MOCK-infected, and overexpressing U937 AML cells treated with cytarabine (WT vs. OE $P<0.0001$; MOCK vs. OE $P<0.0001$ ). c Growth inhibition of wild-type, MOCK-infected, and overexpressing U937 cells treated with cytarabine at the IC 50 of $4 \mu \mathrm{M}$ (WT vs. OE $P<0.005$; MOCK vs. OE $P<0.005$ ). d, e Percent apoptosis of MOCK-infected and overexpressing U937 AML cells treated with cytarabine by FACS analysis (e, OE vs. MOCK $P<0.0001)$. f Representative histograms of cell cycle phases in MOCK-infected and overexpressing U937 cells by FACS analysis. $\mathbf{g}$ The tumor tissues from the xenograft mouse model injected with wild-type (MOCK) and hsa-miR-12462 overexpressing U937 cells (OE). h, i Tumor weights (h) and sizes (i) from mice injected with wild-type and overexpressing U937 cells (MOCK vs. OE $1.11380 \pm 0.27820 \mathrm{~g}$ vs. $0.16000 \pm 0.02266 \mathrm{~g}, n=6, P=0.0057$ ). j Axial anatomic image of mice injected with overexpressing and wild-type U937 cells obtained with 3.0-T MRI. FOV $=64 \mathrm{~mm}$

controls (Fig. 1b, c, Figure S1F-G). The apoptosis rate of the hsa-miR-12462 overexpressing cells was significantly higher than that of the control (Fig. 1d, e, Figure S1I,L) as well. These differences were not seen in U937 cells (Figure S1H,J) or HL-60 cells (Figure S1K) treated with doxorubicin. We hypothesized that these different 
responses might reflect the effects of hsa-miR-12462 on the cell cycle. Cell cycle analysis revealed that a greater proportion of hsa-miR-12462-expressing cells were adjusted in $G_{0} / G_{1}$ and $S$-phase when compared with MOCK-transfected cells (Fig. 1f, Figure S1M).

We further studied the biological behavior of the hsa-miR12462 overexpressing U937 cells in a subcutaneous xenograft mouse model (Fig. 1g). Tumor weight and size in the overexpressing cohort were decreased when compared with those of the MOCK-transfected cohort (Fig. 1h, i). (MOCK vs. OE: $P=0.0057)$. These differences were further confirmed by both magnetic resonance imaging (MRI; Fig. 1j) and histopathology (Figure S1N-P).

Through RNA-sequencing analysis of hsa-miR-12462 overexpressing and MOCK-transfected U937 cells, 306 genes were identified with differential expressions (Fig. 2a, S2A). Gene Ontology (GO) and Kyoto Encyclopedia of Genes and Genomes (KEGG) pathway enrichment analyses were done as well (Figure S2B-C). Enrichment analysis of the KEGG pathway indicates the involvement of the cAMP signaling pathway (Figure S2C) [7]. In addition, CCNE1, E2F4, and TP53 involved in cell cycle regulation

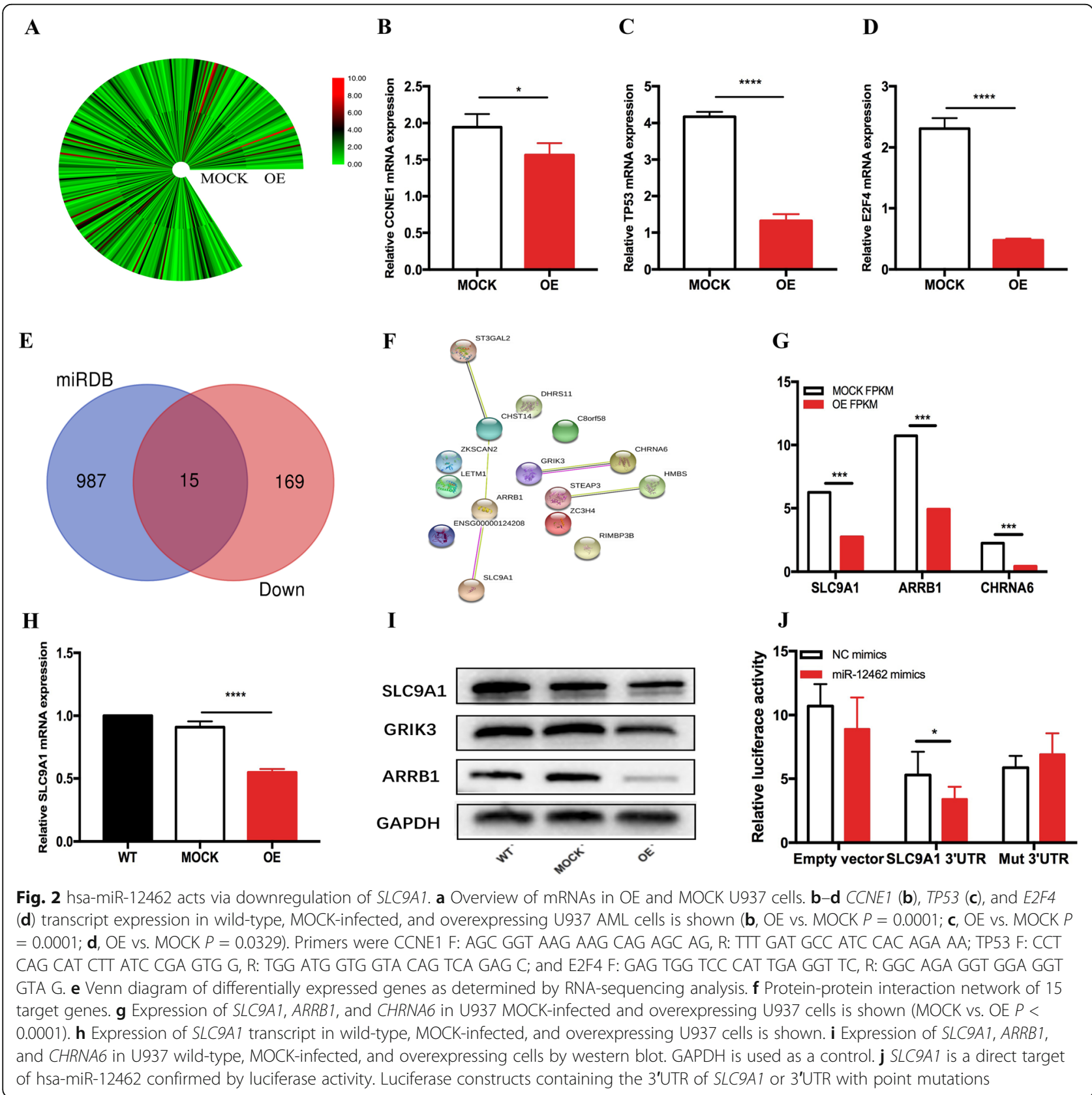


were found significantly downregulated in the RNAsequencing analysis (Figure S2A). Q-RT-PCR analysis of these genes confirmed the results from the RNAsequencing analysis (Fig. 2b-d). Combining the data from the MiRDB, an online microRNA database (http://mirdb. org) [8], with the RNA-sequencing results, 15 genes were found to share predicted targets (Fig. 2e, f) including SLC9A1, ARRB1, and CHRNA6 (Fig. 2g). SLC9A1 (NHE1), the most common isomer in the $\mathrm{Na}^{+} / \mathrm{H}^{+}$exchanger family [9], is important in cell transformation [10]. $\beta$ arrestins (ARRBs) participate in mediating tumor proliferation and inflammation-induced cancer development [11], whereas nicotinic acetylcholine receptors (CHRNs) are important regulators of tobacco-induced carcinogenesis [12]. The mRNA and protein levels of SLC9A1, ARRB1, and CHRNA6 were inhibited by overexpression of hsamiR-12462 (Fig. 2h, i, S2D-E). Using a luciferase 3'UTR reporter assay, we found that hsa-miR-12462 bound exclusively to the 3'UTR of SLC9A1 in U937 cells (Fig. 2j; S2F-G). In summary, a higher level of hsa-miR-12462 in AML cells is associated with increased sensitivity to cytarabine chemotherapy via downregulation of SLC9A1.

\section{Supplementary information}

Supplementary information accompanies this paper at https://doi.org/10. 1186/s13045-020-00935-w.

Additional file 1: Table S1. Patient information. Table S2.

Demographic and AML - related features of the expression level of hsamiR-12462

Additional file 2: Figure S1. Hsa-miR-12462 inhibits growth of AML both in cell lines and animal models. A.Schematic diagram of the secondary structure of mature hsa-miR-12462.Structure was predicted by MFOLD. B. hsa-miR-12462 transcript expression in Wild-type, MOCKinfected, and Over-expressing U937 cells (OE vs. MOCK P = 0.0005). C. Growth rates of HL-60 Wild-type, MOCK-infected and Over-expressing cells (OE vs. MOCK P < 0.0001). D-E. Cell growth of MOCK and Overexpressing U937 OE vs. MOCK P $=0.0004$ ) cells as measured by EdU. F. Growth inhibition of Wild-type, MOCK infected and Over-expressing $\mathrm{HL}$ 60 cells treated with cytarabine(OE vs. MOCK P=0.0428). G. Growth inhibition of Wild-type, MOCK-infected, and Over-expressing HL-60 cells treated with cytarabine at the $\mathrm{IC}_{50}=2.9 \mu \mathrm{M}$ (MOCK vs. OE P = 0.0042). $\mathrm{H}$ . Growth inhibition rates of Wild-type, MOCK-infected and Overexpressing U937 cells treated with doxorubicin $\mathrm{IC}_{50}=6 \mu \mathrm{M}$ (OE vs. MOCK vs. WT P > 0.05). I,L. Percent apoptosis of MOCK infected and Overexpressing $\mathrm{HL}-60$ cells treated with cytarabine by FACS analysis ( $\mathrm{L}, \mathrm{OE}$ vs. MOCK P $=0.0377$ ). J,K: Apoptosis percentage of MOCK-infected and Overexpressing $\mathrm{U} 937(\mathrm{~J})$ and $\mathrm{HL}-60(\mathrm{~K})$ cells treated with doxorubicin by FACS analysis. ( J.OE vs. MOCK P > 0.05.K:OE vs. MOCK P > 0.05) M. Quantification of cell-cycle phases in MOCK-infected and over-expressing U937 cells by FACS analysis. N. Hematoxylin and eosin (HE) staining and immune histochemistry analyses of hsa-miR-12462 OE and MOCK tumor sections. HE staining magnification: $\times 20$. MPO staining magnification: $\times 20$. Ki-67 staining magnification: $\times 20.0-P$. Percentage of myeloperoxidase (MPO) and Ki-67 expression in OE and MOCK U937 cells (MOCK vs. OE: O, P< 0.0001. $P, P<0.0001)$

Additional file 3: Figure S2. The downstream targets of hsa-miR12462. A. Differential expression of selected genes in hsa-miR-12462 OE and MOCK U937cells. B-C. (B) GO and (C) KEGG pathway enrichment analysis of differentially expressed genes after RNA-sequencing in U937 OE/ MOCK cells. D-E. Transcript expression of CHRNA6 (D) and ARRB1 (E) in wild-type, MOCK-infected and OE U937 cells is shown. F-G. Luciferase reporter assay showing the 3'-UTR segments of (F) ARRB1 and (G) CHRN A6 do not contain hsa-miR-12462 binding sites. Error bars indicate \pm SD; $\mathrm{n}=3$ each. $\mathrm{P}$ values were obtained by the two-tailed Student t-test.

Additional file 4. Detailed materials and methods

\section{Abbreviations}

AML: Acute myeloid leukemia; SLC9A1: Solute carrier family 9 member A1; CAMP: Cyclic adenosine monophosphate; CCNE1: CyclinE1; E2F2: E2F transcription factor 2; ARRB1: Arrestin beta-1; CHRNA6: Cholinergic receptor, nicotinic, alpha 6

\section{Acknowledgements}

We thank Dr. Yang-Qiu Li, Robert Peter Gale, Wei Liu, Christopher Louis Robinson, and You-Er He for providing helpful comments.

\section{Authors' contributions}

$\mathrm{HZ}$ designed the study; YJ and WL performed major experiments; HEZ, XPY, $H L, Q L Z, X Y J, H Y Z$, and $L Z$ performed the rest of the necessary experiments; $X L Z$ provided some clinical samples and also gave some useful suggestions. $\mathrm{YJ}, \mathrm{WL}$, and $\mathrm{HZ}$ wrote and edited the manuscript, and $\mathrm{HZ}$ approved the final manuscript. The authors read and approved the final manuscript.

\section{Funding}

The project is supported by the National Natural Science Foundation of China (Grant Nos. 81770184 and 81970143).

\section{Availability of data and materials}

All supporting data are included in the manuscript and supplemental files. Additional data are available upon reasonable request to the corresponding author.

Ethics approval and consent to participate

This study was approved by the Ethics Committee of Xiangya Hospital, Central South University, in China, and all subjects gave written informed consent.

Consent for publication

Not applicable.

\section{Competing interests}

The authors declare that they have no competing interests.

\section{Author details}

${ }^{1}$ Department of Hematology, Xiangya Hospital, Central South University, Changsha, Hunan, China. ${ }^{2}$ Department of Hematology, The First Affiliated Hospital of Jinan University, Guangzhou, Guangdong, China. ${ }^{3}$ Department of Radiology, Xiangya Hospital, Central South University, Changsha, Hunan, China. ${ }^{4}$ Department of Pathology, Xiangya Hospital, Central South University, Changsha, Hunan, China.

Received: 27 May 2020 Accepted: 13 July 2020

Published online: 23 July 2020

\section{References}

1. Guttman M, Rinn JL. Modular regulatory principles of large non-coding RNAs. Nature. 2012;482(7385):339-46.

2. Liu Y, Cheng Z, Pang Y, Cui L, Qian T, Quan L, Zhao H, Shi J, Ke X, Fu L. Role of microRNAs, circRNAs and long noncoding RNAs in acute myeloid leukemia. J Hematol Oncol. 2019;12(1):51.

3. Wang WT, Han C, Sun YM, Chen TQ, Chen YQ. Noncoding RNAs in cancer therapy resistance and targeted drug development. J Hematol Oncol. 2019; 12(1):55.

4. Mishra PJ. MicroRNAs as promising biomarkers in cancer diagnostics. Biomark Res. 2014:2:19.

5. Niparuck $P$, Limsuwanachot $N$, Pukiat $S$, Chantrathammachart $P$, Rerkamnuaychoke B, Magmuang S, Phusanti S, Boonyawat K, Puavilai T, Angchaisuksiri $P$, et al. Cytogenetics and FLT3-ITD mutation predict clinical outcomes in non transplant patients with acute myeloid leukemia. Exp Hematol Oncol. 2019;8:3. 
6. de Rie D, Abugessaisa I, Alam T, Arner E, Arner P, Ashoor H, Åström G, Babina M, Bertin N, Burroughs AM, et al. An integrated expression atlas of miRNAs and their promoters in human and mouse. Nature biotechnology. 2017:35(9):872-8.

7. Park JY, Juhnn YS. CAMP signaling increases histone deacetylase 8 expression via the Epac2-Rap1A-Akt pathway in H1299 lung cancer cells. Exp Mol Med. 2017;49(2):e297.

8. Chen Y. Wang X: miRDB: an online database for prediction of functional microRNA targets. Nucleic Acids Res. 2020;48(D1):D127-d131.

9. Neri D, Supuran $\mathrm{CT}$. Interfering with $\mathrm{pH}$ regulation in tumours as a therapeutic strategy. Nat Rev Drug Discov. 2011;10(10):767-77.

10. Shorthouse D, Riedel A, Kerr E, Pedro L, Bihary D, Samarajiwa S, Martins CP Shields J, Hall BA. Exploring the role of stromal osmoregulation in cancer and disease using executable modelling. Nat Commun. 2018;9(1):3011.

11. Yang Y, Guo Y, Tan S, Ke B, Tao J, Liu H, Jiang J, Chen J, Chen G. Wu B: BArrestin1 enhances hepatocellular carcinogenesis through inflammationmediated Akt signalling. Nat Commun. 2015;6:7369.

12. Grando SA. Connections of nicotine to cancer. Nature reviews Cancer. 2014; 14(6):419-29.

\section{Publisher's Note}

Springer Nature remains neutral with regard to jurisdictional claims in published maps and institutional affiliations.

Ready to submit your research? Choose BMC and benefit from:

- fast, convenient online submission

- thorough peer review by experienced researchers in your field

- rapid publication on acceptance

- support for research data, including large and complex data types

- gold Open Access which fosters wider collaboration and increased citations

- maximum visibility for your research: over $100 \mathrm{M}$ website views per year

At BMC, research is always in progress.

Learn more biomedcentral.com/submissions 